BUCHBESPRECHUNGEN

DOI: $10.1007 / \mathrm{S} 00350-009-2391-0$

\section{Haftung medizinischer Ethik-Kommissionen bei klinischer Arzneimittelprüfung.}

Von Michael van der Sanden. (Düsseldorfer Rechtswissenschaftliche Schriften, Bd. 57). Nomos Verlagsgesellschaft, Baden-Baden 2008, 288 S., kart., $€ 64,00$

Die von Dirk Olzen geförderte Düsseldorfer juristische Dissertation gilt einem Gegenstand, der durch das 12. Gesetz zur Änderung des Arzneimittelgesetzes von 2004 (BGBl. I S. 2031) zusätzliches Gewicht gewonnen hat. Die öffentlich-rechtlich verfassten Ethik-Kommissionen bei den Ärztekammern und bei den Hochschulen haben zur Hauptaufgabe die Begutachtung klinischer Arzneimittelprüfungen, die der Forschungsfreiheit des Studienbetreibers einerseits, wie dem gleichfalls grundgesetzlich verbrieften Schutz der Autonomie und körperlichen Integrität der Versuchspersonen andererseits genügen müssen. Die Verdichtung des Netzes von Normen mit vermehrten Verfahrensvorgaben und die Aufwertung der Kommissionsvoten machen die Aufgabe noch anspruchsvoller. Wo Sorgfaltspflichten wachsen, erhöht sich das Haftungsrisiko, hier im Verhältnis zu Studienteilnehmern und zu Sponsoren. Dabei geht es um die Verarbeitung einer Fülle von Informationen in einem eng geschnürten Fristenkorsett.

Der Autor erörtert die einschlägigen Fragen umfassend, umsichtig und abgewogen. So sieht er im Kommissionsvotum wohlbegründet einen Verwaltungsakt, wobei freilich auch die Bedenken erscheinen (S. $47 \mathrm{ff}$.). Die Einstandspflicht der Ethik-Kommission gegenüber dem Versuchsteilnehmer (unabhängig davon, ob er das Testpräparat, die Standardtherapie oder ein Placebo erhält) wie dem Sponsor bestimmt sich ausschließlich nach den Grundsätzen der Amtshaftung nach $\$ 839$ BGB i.V. mit Art. 34 GG. Die den Amtshaftungsanspruch auslösende Verletzung der Begutachtungspflicht liege in der Stimmabgabe eines jeden einzelnen fehlerhaft votierenden Kommissionsmitglieds, sofern sich dessen Meinung in der Kommissionsentscheidung realisierte. Die dafür erforderliche Zurechnung der übrigen Stimmen erfolge über $₫ 830$ Abs. 1 S. 1 BGB, durch dessen Anwendung auch der Ausschluss des Einwandes rechtmäßigen Alternativerhaltens gelinge - ein interessanter Gedanke (S. $107 \mathrm{ff}$.). Die Subsidiaritätsklausel (\$839 Abs. 1 S. 2 BGB) entlastet den Kommissionsträger in Fällen der Haftungskonkurrenz insbesondere bei Ansprüchen gegen private Forschungsinstitutionen oder gegen Sponsoren. Das Haftpflichtrisiko gegenüber Versuchsteilnehmern mindert die Probandenversicherung, die verschuldensunabhängig regelmäßig einen großen Teil der Risiken auffängt. Weil der Ethik-Kommission Ermessens- und Beurteilungsspielräume zu Gebote stehen und die richterliche Kontrolle darum einer gewissen Begrenztheit unterliegt, mag auch daraus eine Mäßigung des Haftpflichtrisikos folgen. Beteiligten Ethik-Kommissionen bleibt nur knapp bemessene Zeit. „Die Bewertung der über das Pflichtprogramm des $\$ 8$ Abs. $5 \mathrm{~S} .2$ GCP-Verordnung hinausgehenden Kriterien wird sich angesichts dessen regelmäßig auf die Beachtung allgemeiner Standards und die Kennzeichnung offensichtlicher Defizite beschränken“ (S. 196) Beim vertikalen Ausgleich über den Innenregress bei den Kommissionsmitgliedern kommt das Arbeitsrecht mit der Fürsorgepflicht des Rechtsträgers ins Spiel, wobei sich freilich eine verlässliche und vollständige Entlastung nur durch eine Selbstverpflichtungserklärung des Kommissionsträgers erreichen lässt (S. 259f.). Für die Absicherung der Kommissionsträger empföhle sich, so der Autor, ein Trägermodell wie die verwaltungsorganisatorische Neukonzeption im Land Berlin.

Das Buch empfiehlt sich, weil es kompetent ein Feld bestellt, auf dem forensische Erfahrungen noch weithin fehlen - worüber nicht zu klagen ist - und das als neu eingerichtetes eindringender juristischer Pflege nach Art der geleisteten bedarf.

Prof. Dr. iur. Dr. h.c. Adolf Laufs,

Heidelberg, Deutschland

\section{Die Regulierung der Transplantationsmedizin in Deutschland.}

Herausgegeben von Wolfram Höfling. Verlag Mohr Siebeck, Tübingen 2008, VIII u. 138 S., brosch., $€ 39,00$

Der Sammelband entstand im Anschluss an die „3. Kölner Gespräche zu Recht und Ethik in der Gesundheitsversorgung“, die Anfang 2008 zehn Jahre nach Inkrafttreten des deutschen Transplantationsgesetzes (TPG) - an der Universität zu Köln stattfanden. Thema des Symposions waren denn auch die alten und neuen Probleme im Zusammenhang mit diesem Gesetz, die in den einzelnen von Wolfram Höfling gesammelten und herausgegebenen Beiträgen aus unterschiedlichen Blickwinkeln reflektiert werden.

Der Herausgeber (S. 3-7) leitet den Band mit einer kritischen Zwischenbilanz nach 10 Jahren Transplantationsgesetz ein, in der er zunächst feststellt, dass die Konfliktpunkte, die schon das Gesetzgebungsverfahren geprägt hatten - die Hirntod-Debatte und die Frage nach einer Widerspruchs- oder Zustimmungslösung - nach wie vor aktuell und keineswegs geklärt sind. Das Kölner Symposion, das der vorliegende Band dokumentiert, knüpft an diese Debatte an, war aber vor allem den Problemen gewidmet, die damals in den Hintergrund traten oder zwischenzeitlich erst deutlich geworden sind.

Schwerpunkte sind dabei der nach wie vor bestehende Mangel an Spenderorganen, die als zu restriktiv erachteten Regelungen zur Lebendorganspende und das Regelungsdefizit im Bereich der Organallokation.

Aus Sicht des Transplantationsmediziners Richard Viehbahn (S. 9-26) liegen die Defizite des TPG vor allem in den erstgenannten Bereichen und weniger im Bereich der Organallokation. Für diese gab es bereits frühzeitig kodifizierte Selbstverpflichtungen der an Transplantationen beteiligten Institutionen und aus Sicht des Mediziners scheint es nicht problematisch, dass das TPG daran nichts geändert hat. Da das Problem der Organverteilung hauptsächlich in der Mangelsituation gesehen wird, durch die der aus medizinischer Sicht erfolgversprechenden Therapieform der Transplantation enge Grenzen gesetzt werden, richten sich Viehbahns Verbesserungsvorschläge auf Maßnahmen zur Steigerung des Organaufkommens. Hier wird zunächst eine bessere Information der Bevölkerung gefordert, vorzugsweise in Kombination mit der Einführung einer Widerspruchslösung anstelle der erweiterten Zustimmungslösung. Notwendig erscheint auch eine Förderung der Kooperation der Krankenhäuser bei der Spendererkennung, etwa in Form von Sanktionen für Verstöße gegen die im TPG normierte Meldepflicht.

Außerdem gibt es in Deutschland bislang keine Regelung über sog. marginale Organe (d.h. solche mit eingeschränkter Funktionsfähigkeit) und sog. „,non-heartbeating donors“, also Organe, die erst nach dem Herz-Kreislauf-Tod des Patienten entnommen werden. Im Ausland werden mit solchen Organen bereits Therapieerfolge erzielt, weswegen der Autor hier eine weitere Möglichkeit sieht, dem Organmangel entgegenzuwirken

Bedeutsamer erscheint dem Autor aber die Ausweitung der Möglichkeiten der Lebendspende, für deren restriktive Handhabung es keine medizinischen Gründe gibt. Hierzu gehört auch eine Verbesserung des zum Teil lückenhaften Versicherungsschutzes der Lebendspender und die bevorzugte Behandlung von Spendern, wenn diese aufgrund der Spende später selbst ein Transplantat benötigen.

An dieses Problem knüpft der Beitrag von Stephan Rixen (S. 73-83) an. Der Autor hält zum einen die Begrenzung des Spenderkreises und zum anderen die grundsätzliche Subsidiarität der Lebendspende für verfehlt.

Der vom Gesetzgeber angeführte Grund für diese Beschränkungen, die Freiwilligkeit der Spenderentscheidung sicherstellen zu wollen, trägt nicht, da nicht berücksichtigt wird, dass gerade angesichts des Leidens einer nahestehenden Person großer sozialer oder moralischer Druck auf den potentiellen Spender entstehen kann. Und auch die Begründung, dem verbotenen Organhandel entgegenwirken zu 